\title{
The Effects of a Probiotic (Bacillus clausii) in Acute Kidney Injury in a Rat Model of LPS-Induced Endotoxemia
}

\author{
Asli Kandil' ${ }^{1}$ \\ IIstanbul University, Faculty of Science, Department of Biology, Istanbul, Turkey
}

ORCID IDs of the authors: A.K. 0000-0001-8408-2610

Please cite this article as: Kandil A. The Effects of a Probiotic (Bacillus clausii) in Acute Kidney Injury in a Rat Model of LPSInduced Endotoxemia. Eur J Biol 2021; 80(1): 48-53. DOI: 10.26650/EurJBiol.2021.931755

\section{ABSTRACT}

Objective: This study aims to examine the role of a probiotic bacterium, Bacillus clausii, on oxidative stress in a lipopolysaccharide (LPS)-induced acute kidney injury (AKI) rat model.

Materials and Methods: The rats were divided into four groups: Control group, LPS group (1.5 mg/kg, LPS), Probiotic+LPS group in which LPS was given after administration of Bacillus clausii as a probiotic for 21 days, and Probiotic group. The kidneys of the rats were removed 24 hours after LPS injection. Total antioxidant status, total oxidant status, oxidative stress index, malondialdehyde and myeloperoxidase (MPO) values were biochemically determined in the kidneys. Furthermore, the kidney tissue samples were immunohistochemically stained for interleukin-6 (IL-6) and tumor necrosis factor (TNF)- $a$ expression, and leukocyte distribution.

Results: Endotoxemia caused an increase in oxidative stress $(p<0.001)$, lipid peroxidation $(p<0.01)$, MPO activity $(p<0.001)$, and the expression of IL-6 $(p<0.001)$ and TNF- $a(p<0.001)$. The administration of probiotic ameliorated oxidative stress, lipid peroxidation, and myeloperoxidase activity, and resulted in decreased IL- 6 and TNF-a reactions that were elevated with LPS treatment.

Conclusion: The results suggest that Bacillus clausii as a probiotic bacterium may have an antioxidative property in LPSinduced AKI.

Keywords: Probiotic, acute kidney injury, endotoxemia, oxidative stress, Bacillus clausii

\section{INTRODUCTION}

Microbiota consists of various microorganisms (bacteria, fungi, and protozoa) (1). There is a symbiotic and mutualistic relationship between microorganisms in the gut microbiota and host (2). In mammals, the microbiota is composed mainly of four main phyla of eubacteria: Bacteroidetes, Firmicutes, Actinobacteria, and Proteobacteria $(3,4)$. The microbiota plays significant roles in various physiological events, such as protection against infection, recovery from disease, drug metabolism, nutritional status, and vitamin synthesis $(3,5)$.

The change in gut microbiota is called dysbiosis, leading to a disrupted interaction between host and microbes. The resulting systemic complications and disease may follow deterioration in microbiota homeostasis caused by excessive increase or depletion of specific bacterial species (6). Dysbiosis relates to several diseases such as obesity, diabetes, and inflammatory bowel and critical disease $(7,8)$. It was shown that the loss of "health promoting" bacteria and dysbiosis could contribute to infections, organ failure, and sepsis in the intensive care unit (ICU) (7). Some members of the gut microbiota generate anti-inflammatory molecules and proteins, while the microbiota can cause inflammatory responses. It is thought that the loss of useful microorganisms can disrupt nutrition in humans and trigger inflammatory responses. Hence, modification of the 
microbiota composition can restore nutritional deficiencies and anti-inflammatory effects (9).

Probiotics have attracted attention as an opportunity to treat and prevent different diseases such as diabetes, obesity, allergies, and infections (10-12). In recent years, the question has been raised as to whether restoration of ICU patients through probiotics or synbiotics to interfere with the preservation and degradation of the microbiota would be an optimal interference to prevent infection and improve recovery.

The supplementation of probiotics offers a significant approach to critical illness therapy because of the important roles of microbiota in the modulation of nutrient production and absorption, and inflammation (9). Probiotics are live microorganisms which, when they are administered in adequate amounts, confer a health benefit on the host (13).

Different dietary supplements, functional foods, or probiotic strains that can be beneficial for human health have been designed. Data obtained from studies have supported the use of bacterial spore formers as probiotics and food supplements (14).

Bacillus spp., one of the spore-forming probiotics, are Gram-positive, aerobic or facultative anaerobic bacteria, and can survive in heat, acidic stomach, and other extreme environmental conditions (15-17). Therefore, spores of Bacillus spp. are generally used as probiotic preparations for different purposes (18).

Acute kidney injury (AKI) is a serious and common complication of sepsis in ICU patients (19). The pathophysiology of AKI in endotoxemic shock or sepsis is complex, and contains hemodynamic changes, inflammation, and damage. Moreover, sepsis-induced immune responses include the activation of both pro-inflammatory and anti-inflammatory events $(20,21)$. In the literature, it has been shown that the gut microbiota plays a role in kidney disease (22-25). Although the function of gut microbiota has been determined in kidney disease, the relationship between AKI and microbiota is not yet fully understood.

This study aimed to investigate the effects of the probiotic bacterium Bacillus clausii on renal oxidative stress in a lipopolysaccharide (LPS)-induced AKI model.

\section{MATERIALS AND METHODS}

\section{Animals and Experimental Protocol}

The experiments were designed with 27 male Wistar albino rats (3 months old, 300-400 g) supplied from Bezmiâlem Vakıf University, Istanbul, Turkey. The animals were allowed free access to a pelleted diet and tap water. The study was approved and reviewed by Bezmiâlem Vakıf University Animal Experiments Local Ethics Committee (Decision no: 2021/54).

The rats were divided into four groups. The control group $(n=6)$ was injected intraperitoneally (ip) with $0.9 \% \mathrm{NaCl}$ solution (physiological saline) after physiological saline was given by gavage for 21 days. In the LPS group ( $n=7)$, LPS (1.5 mg/kg LPS, ip, E. coli, Serotip 0111: B4, Sigma, Missouri, USA) was injected ip after physiological saline was given by gavage for 21 days. In the Probiotic+LPS group $(n=6)$, LPS was injected ip into animals after the administration of $B$. clausii $\left(1.25 \mathrm{ml}, 1 \times 10^{9} \mathrm{CFU}\right.$ per animal via gavage) as a commercial probiotic (Enterogermina $^{\circledR}$, Sanofi, Turkey) for 21 days. Probiotic group $(n=8)$ was ip treated with physiological saline after B. clausii was given by gavage for 21 days. All applications were done at the same time every day.

The rats were anesthetized 24 hours after LPS injection. The kidney samples were removed for biochemical and immunohistochemical analyses.

\section{Biochemical Analyses}

The tissue samples were homogenized and centrifuged at 3000 rpm for 20 minutes at $+4{ }^{\circ} \mathrm{C}$ (Sorvall Super T21, Benchtop Centrifuge). The supernatants were collected from homogenates.

Total oxidant status (TOS) $\left(\mu \mathrm{mol} \mathrm{H}_{2} \mathrm{O}_{2}\right.$ eq/L), total antioxidant status (TAS) (mmol Trolox eq/L), and oxidative stress index (OSI) (arbitrary unit) were measured in the kidney tissues using commercial kits (Rel Assay Diagnostics; Gaziantep, Turkey).

Malondialdehyde (MDA) in the kidney was measured using the spectrophotometric method (26). The results were expressed as $\mathrm{nmol} / \mathrm{mg}$ protein.

Myeloperoxidase (MPO) activity was measured according to the method of Krawisz et al. (1984) (27). The results were expressed as $\mu \mathrm{mol} / \mathrm{mL}$.

Protein levels in homogenate/postmitochondrial fractions were measured using the bicinchoninic acid reaction (28).

\section{Immunohistochemical Analyses}

The kidney samples were fixed in a neutral buffer formaldehyde solution, and histologically processed as previously described (29). The kidney sections ( $5 \mu \mathrm{m}$ thick) were stained with tumor necrosis factor (TNF)-a (diluted 1/200) (Abcam ab66579), interleukin-6 (IL-6) (diluted 1/200) (Abcam, 6672), and MPO (diluted 1/75) (Thermo Fisher Scientific, RB-373-A) antibodies. The antibody staining was scored as previously described by Legrand et al. (29).

\section{Statistical Analysis}

Significant differences between groups were estimated using one-way ANOVA with Tukey's post-test using GraphPad Prism version 5.0 for Windows (GraphPad Software, San Diego, CA, USA). A value $p<0.05$ was considered statistically significant. The results were expressed as the mean \pm SEM.

\section{RESULTS}

\section{Biochemical Results}

TOS levels increased in renal tissues of LPS-injected animals $(p<0.001)$, and diminished in the Probiotic+LPS group, but was high with respect to the control $(p<0.001)$. In the Probiotic group, TOS levels elevated according to the control $(p<0.05)$ 
A

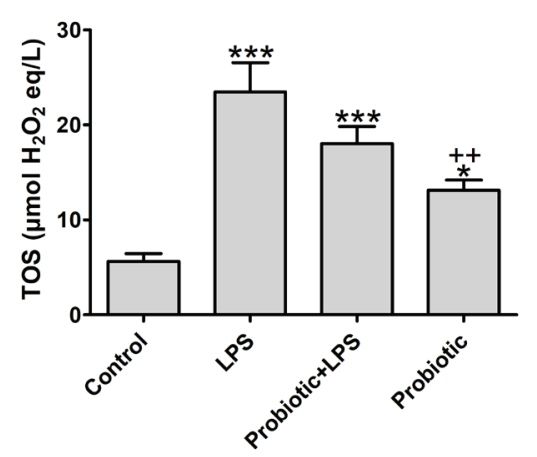

B

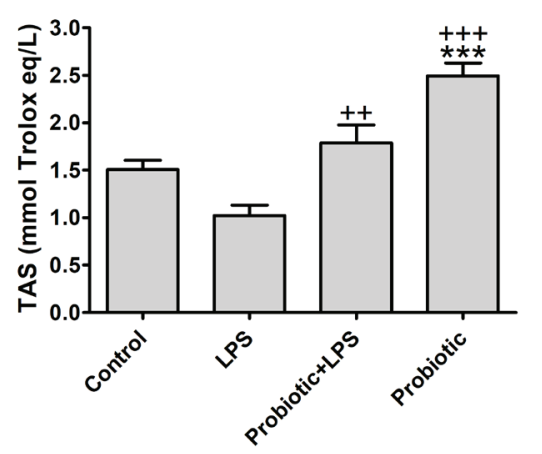

C

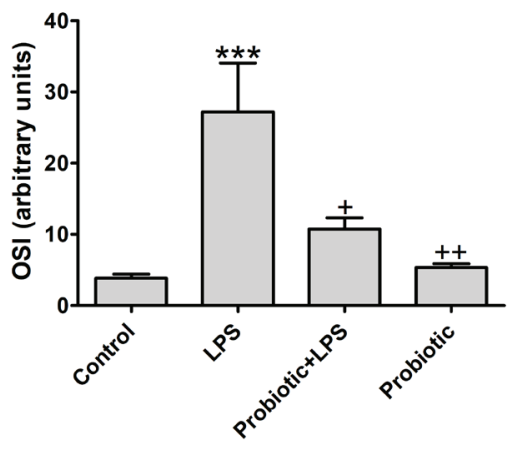

Figure 1. Effects of a probiotic bacterium Bacillus clausii on renal oxidative stress in lipopolysaccharide (LPS)-induced endotoxemia. (A) Kidney total oxidant status (TOS), (B) total antioxidant status (TAS) and (C) oxidative stress index (OSI) values in the experimental groups. ${ }^{* * *} p<0.001,{ }^{*} p<0.05$ vs. Control group. ${ }^{+++} p<0.001,{ }^{++} p<0.01,{ }^{+} p<0.05$ vs. LPS group.

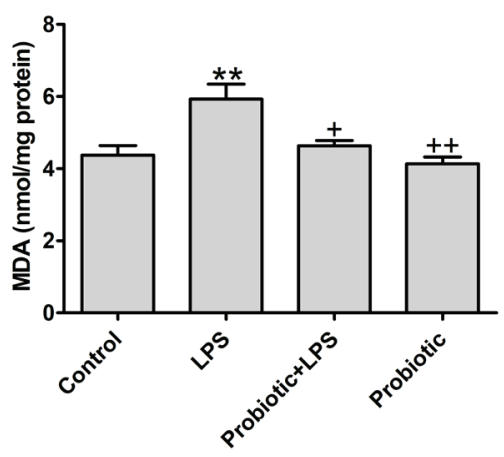

B

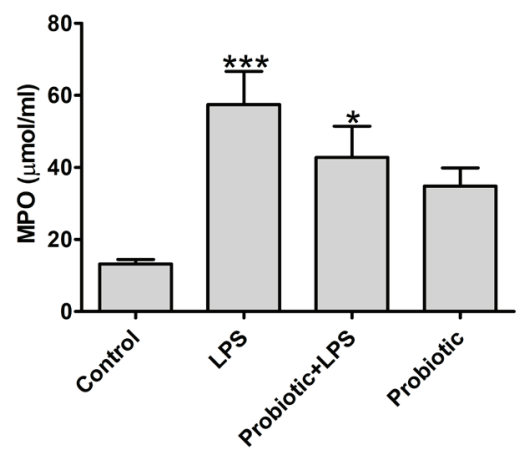

Figure 2. Effects of a probiotic bacterium Bacillus clausii on kidney malondialdehyde (MDA) levels (A) and myeloperoxidase (MPO) activity (B) in lipopolysaccharide (LPS)-induced endotoxemia. ${ }^{* * *} p<0.001,{ }^{* *} p<0.01,{ }^{*} p<0.05$ vs. Control group. ${ }^{++} p<0.01,{ }^{+} p<0.05$ vs. LPS group.

(Figure 1A). TAS levels reduced in renal tissues of the LPS group. Administration of the probiotic caused an increase in TAS levels $(p<0.01)$ in the Probiotic+LPS group. Moreover, TAS level was similar to the control group. TAS levels increased in the Probiotic group compared with the control group $(p<0.001)$ (Figure 1B). OSI values increased $(p<0.001)$ in the LPS group compared with the control group. In the Probiotic+LPS group, OSI values decreased compared with the LPS group $(p<0.05)$. OSI values of probiotic treated groups were similar to the control (Figure 1C).

MDA levels increased in LPS-treated animals $(p<0.01)$ and reduced in the Probiotic+LPS group $(p<0.05)$ when compared to this group. MDA levels of the Probiotic+LPS and Probiotic groups resembled the control group (Figure $2 \mathrm{~A}$ ). MPO activity elevated in the LPS group $(p<0.001)$ and diminished in the Probiotic+LPS group when compared to this group. In the Probiotic+LPS group, it was high with respect to the control group $(p<0.01)$. MPO activity of the Probiotic group was higher than the control group, but it was not statistically significant (Figure 2B).

\section{Immunohistochemical Results}

IL-6 and TNF-a reaction and MPO-stained leukocytes are presented in Figure 3. LPS-induced endotoxemia caused an increase in TNF- $\alpha$ and IL- 6 reactions in the kidney tissues $(p<0.001)$. The probiotic administration decreased IL-6 $(p<0.001)$ and TNF-a reaction $(p<0.01)$ compared with the LPS group. The distribution of MPO-stained leukocytes was marked in both glomeruli and peritubular areas in the LPS groups $(p<0.001)$ in comparison to the control group. The probiotic did not affect the distribution of MPO-stained leukocytes in the kidney tissue in LPS given animals (Figure 3).

\section{DISCUSSION}

AKI is an important health problem (19). Sepsis, endotoxemia, ischemia reperfusion, or nephrotoxins cause AKI (30). The fundamental interaction between kidney and gut microbiota is an important regulating factor in $\operatorname{AKI}(22,23)$. Gut microbes are associated with physiological and pathophysiological processes 

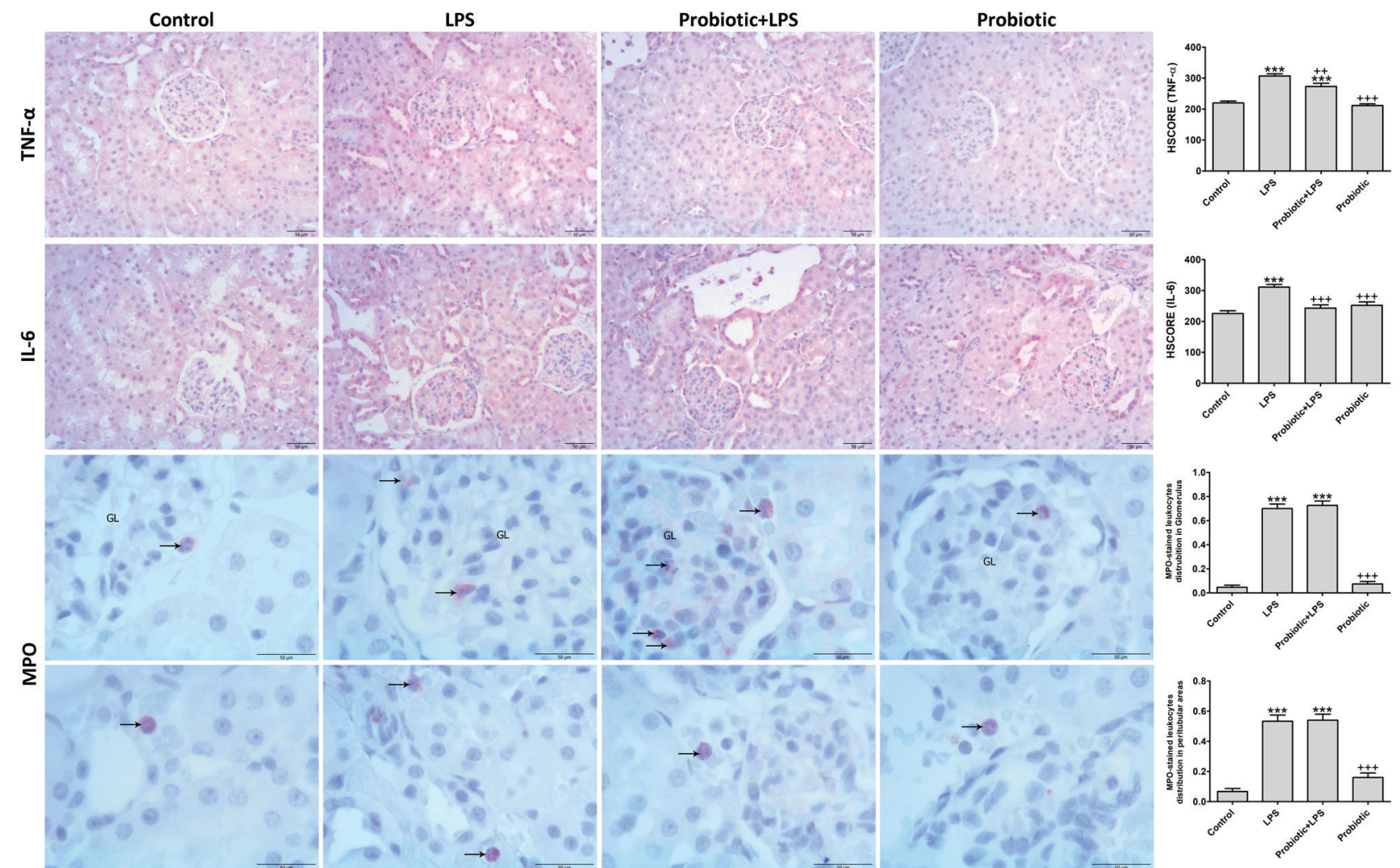

Figure 3. The immunoreactivity and distribution of tumor necrosis factor (TNF)-a, interleukin-6 (IL-6), and myeloperoxidase (MPO)stained leukocytes $(\rightarrow)$ in kidney sections, and HSCORE values of immunoreactive reactions in the experimental groups. Bar: $50 \mu \mathrm{m}$. LPS: lipopolysaccharide. ${ }^{* * *} p<0.001$ vs. Control group. ${ }^{+++} p<0.001,{ }^{++} p<0.01$ vs. LPS group.

in the kidneys (23). Short chain fatty acids (SCFAs) that gut microbes produce improved sepsis-induced AKI (24) and reduced kidney damage and levels of reactive oxygen species and cytokines after kidney ischemia-reperfusion injury (25). Renal structural injury and functional decline following ischemia-reperfusion injury were more severe in germ-free mice compared with control mice (31). It was reported that severely abnormal gut microbiota in patients with renal failure led to aggravate kidney disease (32). Protecting the balance of the gut microbiota was stated to be important for ameliorating dysbiosis that results in immunological dysfunction, inflammation, and renal disease (23). Although the function of gut microbiota has been determined in kidney disease, the link between AKI and microbiota is not yet fully understood. Major advances in studies of the composition and functions of gut microbiota have opened up the opportunity for application of treatment strategies for AKI patients (22).

Changes in gut microbiota control metabolic endotoxemia characterized by translocation of lipopolysaccharides in systemic circulation, and induction of oxidative stress and inflammation in obesity and diabetes (12). Ávila et al. suggest that probiotics and fecal microbiota transplantation could present immunomodulatory advances and may be functional for treatment of pediatric septic patients (33). The different probiotics could reduce the oxidative damage and inflammation in intestines of animals with sepsis (33). Probiotics prevent intestinal and liver damage in sepsis induced with administration of LPS and D-galactosamine (11). The selected probiotic isolates reduced the LPS-caused inflammation in murine macrophage cell line (34). Lactobacillus salivarius reduced inflammation and oxidative stress in cisplatin-induced AKI. These effects were partially mediated by modulating the gut environment (35). In a rat study of sepsis that was induced by injection of LPS after the administration of Lactobacillus rhamnosus $g g$ as probiotic ( $1 \times 10^{7} \mathrm{CFU} /$ day) for 10 days, the probiotic was revealed to have exerted protective effects (36). Similarly, LPS induced oxidative stress and inflammation in the present study. Moreover, the pre-treatment with a probiotic prevented oxidative stress, and exerted protective effects in endotoxemia that was induced by LPS. The probiotic administration after LPS treatment induced a humoral immune response by increasing the levels of immunoglobulins $E, A, G$, and $M$ (37).

A number of Bacillus strains as probiotic have been studied for their potential functions in vivo and in vitro models $(38,39)$. B. clausii is an endospore-forming, gram-positive, facultative alkaliphilic rod bacterium (15), and is able to grow under aerobic and anaerobic conditions and survive gastric acidity $(16,17)$. The role of the Bacillus species ranges from the probiotic nature 
of B. clausii, B. subtilis, B. pumilus, B. coagulans and other strains, to biological control agents ( $B$. sphaericus and $B$. thuringiensis) and pathogenicity (B. cereus and $B$. anthracis) (18). The effects of $B$. clausii have been associated with antimicrobial and immunomodulatory properties (40). B. clausii had a role in production of pro-inflammatory and anti-inflammatory cytokines (41). Furthermore, B. clausii strains release antimicrobial substances (40). The probiotic $B$. clausii strain inhibits the cytotoxic effects caused by $B$. cereus and Clostridium difficile toxins (38). B. clausii may reveal immunomodulating activity in nasal lavage of allergic children (10). B. clausii inhibited secretion of pro-inflammatory cytokines and reactive oxygen species production in rotavirus-infected cells (39). It was demonstrated that pretreatment with probiotic capsules of Bacillus species spores caused a significant decrement in pro-inflammatory cytokines (42). Treatment with $B$. clausii UBBC07 importantly attenuated oxidative stress by increasing catalase and SOD, and decreasing MDA in acetaminophen-induced uremia in rats (43). In our study, the treatment with $B$. clausii importantly ameliorated the oxidative stress and lipid peroxidation caused by LPS injection.

Current data showed that Enterogermina, a spore-based probiotic formulation of Bacillus spp., is a trusted probiotic to manage acute diarrhea and intestinal infections (44) and to decrease anti-Helicobacter pylori treatment $(45,46)$, and exhibits immunomodulatory and antimicrobial activities $(10,40)$.

In the study, the effects of probiotic B. clausii, marketed as Enterogermina ${ }^{\circledast}$, on rat kidneys were examined in LPS-induced AKI. Similar to current data, the present study indicated that probiotic B. clausii has antioxidant and anti-inflammatory properties. The results revealed that LPS gave rise to increase TOS and decrease TAS. It can be said that LPS-induced endotoxemia caused oxidative stress as indicated with an increase in the oxidative stress index. Furthermore, it was found that endotoxemia increased the levels of lipid peroxidation and inflammation in the kidney tissue. In addition, expression of pro-inflammatory cytokines such as TNF- $a$ and IL- 6 and leukocytes infiltration was determined in kidney tissues of endotoxemic rats. The pre-treatment with $B$. clausii ameliorated the oxidative stress, lipid peroxidation, and inflammation, and decreased expression of pro-inflammatory cytokines in LPS-induced endotoxemic rats. However, probiotic treatment did not affect the distribution of leukocytes in kidney tissues.

\section{CONCLUSION}

Our results suggested that probiotic $B$. clausii may have an antioxidative and anti-inflammatory property in LPS-induced AKI. Therefore, the present study supports the efficacy of $B$. clausii in the attenuation of LPS-induced AKI. Nevertheless, further studies should be performed to determine the exact role and effect mechanisms of this probiotic in kidney disease.

Peer-review: Externally peer-reviewed.

Conflict of Interest: The author declares that she has no conflicts of interest to disclose.
Financial Disclosure: There are no funders to report for this submission.

\section{REFERENCES}

1. Cani PD. Human gut microbiome: hopes, threats and promises. Gut 2018; 67(9): 1716-25.

2. Eloe-Fadrosh EA, Rasko DA. The human microbiome: from symbiosis to pathogenesis. Annu Rev Med 2013; 64: 145-63.

3. Turnbaugh PJ, Ley RE, Hamady M, Fraser-Liggett CM, Knight R, Gordon Jl. The human microbiome project. Nature 2007; 449(7164): 804-10.

4. Rinninella E, Raoul P, Cintoni M, Franceschi F, Miggiano GAD, Gasbarrini A, et al. What is the healthy gut microbiota composition? A changing ecosystem across age, environment, diet, and diseases. Microorganisms 2019; 7(1): 14.

5. Levy M, Blacher E, Elinav E. Microbiome, metabolites and host immunity. Curr Opin Microbiol 2017; 35: 8-15.

6. Petersen C, Round JL. Defining dysbiosis and its influence on host immunity and disease. Cell Microbiol 2014; 16(7): 1024-33.

7. Wischmeyer PE, McDonald D, Knight R. Role of the microbiome, probiotics, and 'dysbiosis therapy' in critical illness. Curr Opin Crit Care 2016; 22(4): 347-53.

8. Kho ZY, Lal SK. The human gut microbiome - A potential controller of wellness and disease. Front Microbiol 2018; 9: 1835.

9. Davison JM, Wischmeyer PE. Probiotic and synbiotic therapy in the critically ill: State of the art. Nutrition 2019; 59: 29-36.

10. Ciprandi G, Tosca MA, Milanese M, Caligo G, Ricca V. Cytokines evaluation in nasal lavage of allergic children after Bacillus clausii administration: a pilot study. Pediatr Allergy Immunol 2004; 15(2): 148-51.

11. Ewaschuk J, Endersby R, Thiel D, Diaz H, Backer J, Ma M, et al. Probiotic bacteria prevent hepatic damage and maintain colonic barrier function in a mouse model of sepsis. Hepatology 2007; 46(3): 841-50.

12. Cani PD, Bibiloni R, Knauf $C$, Waget $A$, Neyrinck AM, Delzenne $\mathrm{NM}$, et al. Changes in gut microbiota control metabolic endotoxemia-induced inflammation in high-fat diet-induced obesity and diabetes in mice. Diabetes 2008; 57(6): 1470-81.

13. FAO/WHO working group report on drafting guidelines for the evaluation of probiotics in food London, Ontario, Canada, April 30 and May 1, 2002.

14. Elshaghabee FMF, Rokana N, Gulhane RD, Sharma C, Panwar H. Bacillus as potential probiotics: status, concerns, and future perspectives. Front Microbiol 2017; 8: 1490.

15. Cenci G, Trotta F, Caldini G. Tolerance to challenges miming gastrointestinal transit by spores and vegetative cells of Bacillus clausii. J Appl Microbiol 2006; 101(6): 1208-15.

16. Jenson I., Encyclopedia of food microbiology (Second Edition), Editor(s): Carl A. Batt, Mary Lou Tortorello, Bacillus, Introduction, London, Academic Press, 2014, Pages 111-7.

17. Delbrassinne L., Mahillon J., Encyclopedia of food and health, Editor(s): Benjamin Caballero, Paul M. Finglas, Fidel Toldrá, Bacillus: Occurrence, Academic Press, 2016, Pages 307-11.

18. Khatri I, Sharma G, Subramanian S. Composite genome sequence of Bacillus clausii, a probiotic commercially available as Enterogermina ${ }^{\oplus}$, and insights into its probiotic properties. BMC Microbiol 2019; 19(1): 307 .

19. Hoste EA, Bagshaw SM, Bellomo R, Cely CM, Colman R, Cruz DN, et al. Epidemiology of acute kidney injury in critically ill patients: the multinational AKI-EPI study. Intensive Care Med 2015; 41(8): 1411-23.

20. Zarbock A, Gomez H, Kellum JA. Sepsis-induced acute kidney injury revisited: pathophysiology, prevention and future therapies. Curr Opin Crit Care 2014; 20(6): 588-95. 
21. Gómez H, Kellum JA. Sepsis-induced acute kidney injury. Curr Opin Crit Care 2016; 22(6): 546-53.

22. Rabb H, Pluznick J, Noel S. The microbiome and acute kidney injury. Nephron 2018; 140(2): 120-3.

23. Stavropoulou E, Kantartzi K, Tsigalou C, Konstantinidis T, Romanidou G, Voidarou $C$, et al. Focus on the gut-kidney axis in health and disease. Front Med (Lausanne) 2021; 7: 620102.

24. Al-Harbi NO, Nadeem A, Ahmad SF, Alotaibi MR, Al Asmari AF, Alanazi WA, et al. Short chain fatty acid, acetate ameliorates sepsis-induced acute kidney injury by inhibition of NADPH oxidase signaling in T cells. Int Immunopharmacol 2018; 58: 24-31.

25. Andrade-Oliveira V, Amano MT, Correa-Costa M, Castoldi A, Felizardo RJ, de Almeida DC, et al. Gut bacteria products prevent AKI induced by ischemia-reperfusion. J Am Soc Nephrol 2015; 26(8): 1877-88.

26. Ohkawa H, Ohishi N, Yagi K. Assay for lipid peroxides in animal tissues by thiobarbituric acid reaction. Anal Biochem 1979; 95(2): 351-8.

27. Krawisz JE, Sharon P, Stenson WF. Quantitative assay for acute intestinal inflammation based on myeloperoxidase activity. Assessment of inflammation in rat and hamster models. Gastroenterology 1984; 87(6): 1344-50.

28. Smith PK, Krohn Rl, Hermanson GT, Mallia AK, Gartner FH, Provenzano MD, et al. Measurement of protein using bicinchoninic acid. Anal Biochem 1985; 150(1): 76-85.

29. Legrand M, Bezemer R, Kandil A, Demirci C, Payen D, Ince C. The role of renal hypoperfusion in development of renal microcirculatory dysfunction in endotoxemic rats. Intensive Care Med 2011; 37(9): 1534-42.

30. Bonavia A, Singbartl K. A review of the role of immune cells in acute kidney injury. Pediatr Nephrol 2018; 33(10): 1629-39.

31. Jang HR, Gandolfo MT, Ko GJ, Satpute S, Racusen L, Rabb H. Early exposure to germs modifies kidney damage and inflammation after experimental ischemia-reperfusion injury. Am J Physiol Renal Physiol 2009; 297(5): F1457-65.

32. Wang X, Yang S, Li S, Zhao L, Hao Y, Qin J, et al. Aberrant gut microbiota alters host metabolome and impacts renal failure in humans and rodents. Gut 2020; 69(12): 2131-42.

33. Ávila PRM, Michels $M$, Vuolo F, Bilésimo R, Burger $H$, Milioli MVM, et al. Protective effects of fecal microbiota transplantation in sepsis are independent of the modulation of the intestinal flora. Nutrition 2020; 73:110727.

34. Khanna S, Bishnoi M, Kondepudi KK, Shukla G. Isolation, characterization and anti-inflammatory mechanism of probiotics in lipopolysaccharide-stimulated RAW 264.7 macrophages. World J Microbiol Biotechnol 2020; 36(5): 74.
35. Lee TH, Park D, Kim YJ, Lee I, Kim S, Oh CT, et al. Lactobacillus salivarius BP121 prevents cisplatininduced acute kidney injury by inhibition of uremic toxins such as indoxyl sulfate and pcresol sulfate via alleviating dysbiosis. Int J Mol Med 2020; 45(4): 1130-40.

36. Yilmaz M, Erdem AO. The protective role of probiotics in sepsis-induced rats. Ulus Travma Acil Cerrahi Derg 2020; 26(6): 843-6.

37. Kadafi KT, Wibowo S. Differences in systemic humoral immune response among Balb/c mice administered with probiotic, LPS Escherichia coli, and probiotic-LPS E. coli. Iran J Microbiol 2019; 11(4): 294-9.

38. Ripert G, Racedo SM, Elie AM, Jacquot C, Bressollier P, Urdaci MC. Secreted compounds of the probiotic Bacillus clausii strain O/C inhibit the cytotoxic effects induced by Clostridium difficile and Bacillus cereus toxins. Antimicrob Agents Chemother 2016; 60(6): 3445-54.

39. Paparo L, Tripodi L, Bruno C, Pisapia L, Damiano C, Pastore L, et al. Protective action of Bacillus clausii probiotic strains in an in vitro model of Rotavirus infection. Sci Rep 2020; 10(1): 12636.

40. Urdaci MC, Bressollier P, Pinchuk I. Bacillus clausii probiotic strains: antimicrobial and immunomodulatory activities. J Clin Gastroenterol 2004; 38(6 Suppl): S86-90.

41. Di Caro S, Tao H, Grillo A, Franceschi F, Elia C, Zocco MA, et al. Bacillus clausii effect on gene expression pattern in small bowel mucosa using DNA microarray analysis. Eur J Gastroenterol Hepatol 2005; 17(9): 951-60.

42. Neag MA, Catinean A, Muntean DM, Pop MR, Bocsan Cl, Botan EC, et al. Probiotic Bacillus spores protect against acetaminophen induced acute liver injury in rats. Nutrients 2020; 12(3): 632.

43. Patel C, Patel P, Acharya S. Therapeutic prospective of a spore-forming probiotic-Bacillus clausii UBBC07 against acetaminophen-induced uremia in rats. Probiotics Antimicrob Proteins 2020; 12(1): 253-8.

44. Mazza, P. The use of Bacillus subtilis as an antidiarrhoeal microorganism. Bollettino chimico farmaceutico 1994; 133.1: 3-18.

45. Nista EC, Candelli M, Cremonini F, Cazzato IA, Zocco MA, Franceschi F, et al. Bacillus clausii therapy to reduce side-effects of anti-Helicobacter pylori treatment: randomized, double-blind, placebo controlled trial. Aliment Pharmacol Ther 2004; 20(10): 1181-8.

46. Plomer M, lii Perez M, Greifenberg DM. Effect of Bacillus clausii capsules in reducing adverse effects associated with Helicobacter pylori eradication therapy: A randomized, double-blind, controlled trial. Infect Dis Ther 2020; 9(4): 867-78. 